\title{
Exploration of the potential of the radio-detection technique for the detection of high-energy gamma rays
}

R. Dallier ${ }^{* 1,2}$, L. Bondonneau ${ }^{5}$, D. Charrier ${ }^{1,2}$, A. Coffre ${ }^{2}$, S. Corbel ${ }^{2,4}$, L. Denis ${ }^{2}$, C. Dumez-Viou ${ }^{2}$, A. Escudie ${ }^{1}$, D. García-Fernàndez ${ }^{1}$, J. Girard ${ }^{4,3}$, J.M. Grießmeier ${ }^{5,2}$, L.V.E. Koopmans ${ }^{6}$, A. Loh ${ }^{3}$, L. Martin ${ }^{1,2}$, M. Pandey-Pommier ${ }^{2}$, B. Revenu ${ }^{1,2}$, P. Zarka ${ }^{2,3}$

${ }^{1}$ SUBATECH, Institut Mines-Telecom Atlantique - CNRS - Université de Nantes, Nantes, France

${ }^{2}$ Station de radioastronomie de Nançay, CNRS/Observatoire de Paris - PSL - Université d'Orléans/OSUC, Nançay, France

${ }^{3}$ LESIA, Observatoire de Paris, CNRS, PSL, SU/UP/UO, Meudon, France

${ }^{4}$ AIM/CEA Paris-Saclay, Université Paris Diderot, CNRS, Gif-sur-Yvette, France

${ }^{5}$ LPC2E - Université d'Orléans/CNRS, Orléans, France

${ }^{6}$ Kapteyn Astronomical Institute, University of Groningen, Groningen, the Netherlands

E-mail: richard.dallieresubatech.in2p3.fr

The radio detection of atmospheric particle showers initiated by ultra-high energy cosmic rays is today a proven technique that allows to reconstruct their properties (direction of arrival, energy, nature). Among the precursors of this method, the CODALEMA experiment installed at the Nançay radio-astronomy Observatory has contributed to several major technological innovations relevant for the radio detection technique (autonomous triggering, hybrid reconstruction, very wide frequency bands) and its high-performance antennas are also used on the NenuFAR radio telescope in Nançay. We propose to exploit the unique environment of the Nançay Observatory through the CODALEMA experiment and the NenuFAR radio-telescope to explore the possibilities of radio-detection of atmospheric showers initiated by very high energy photons. The central idea is to phase a large set of antennas (several tens) in the direction of known sources emitting gamma (catalogs H.E.S.S., MAGIC, VERITAS, Fermi-LAT ...) to significantly increase the sensitivity of detection and to use the triggering capabilities on ultra fast transients controlled within the framework of CODALEMA. This would make it possible to observe the sources with a useful cycle close to $100 \%$. We present here the intended instrumental scheme as well as the expected signal level and some first results obtained on the observation of cosmic ray air showers with NenuFAR.

36th International Cosmic Ray Conference -ICRC2019-

July 24th - August 1st, 2019

Madison, WI, U.S.A.

${ }^{*}$ Speaker. 


\section{Introduction and motivations}

In parallel with the impressive development of Cerenkov gamma-ray astronomy in the 2000's, the physics of ultra-high energy cosmic rays (UHECR) has also undergone an important evolution, notably with the commissioning of the Pierre Auger observatory in 2004 and the re-birth and the development of a former method of detection and today of observation of atmospheric showers initiated by cosmic rays: the radio detection, whose progresses have been summarised in recent reviews $[1,2]$. The principle is simple: the development of the cascade of charged secondary particles induced in the atmosphere by the arrival of a cosmic ray (atmospheric shower) is accompanied by a brief pulse of electric field ( $\simeq 20 \mathrm{~ns}$ ) which can then be detected by a radio antenna and analysed at high frequencies (from around $10 \mathrm{MHz}$ up to $300 \mathrm{MHz}$ ). Relying on new theoretical approaches $[3,4,5]$, the radiation detection of the atmospheric showers initiated by the UHECR has seen and still sees many flourishing experiments around the world. As a precursor, since 2003 the multidisciplinary scientific collaboration CODALEMA [6] developed on the Nançay radio observatory site a large collection of detectors, intended to study the radio emission of cosmic showers in the energy range from $10^{16}$ to $10^{18} \mathrm{eV}$. The main arrays of CODALEMA in its current version are a square array $(0.4 \times 0.4 \mathrm{~km})$ of 13 particle scintillator counters (surface detector) and a set of 57 so-called "autonomous" crossed dipoles operating between 20 and $200 \mathrm{MHz}$, not interconnected and synchronized by GPS dating, distributed over $1.1 \mathrm{~km}^{2}$. The autonomous station array is purely self-triggered, meaning that each station is independent. Transients coming from cosmic ray air showers are used to build the event based on several station signals, respecting selection criteria offering a large noise rejection factor (more than $99 \%$ ) and a very good efficiency on cosmic ray air shower transient detection [7].

One of the specificities of UHECR detection is that it is impossible to know a priori in which direction to look, and therefore the detection systems, whatever they are, must have maximum angular acceptance and availability in order to cover all directions of arrival. The UHECR radio detection arrays are no exception to this rule. However, radio antenna signals can be combined $a$ priori or a posteriori in a given direction in order to gain in sensitivity of detection (phasing): it is the principle of past and current generations of so-called "digital" radio telescopes. Our idea is therefore to combine the ability to detect atmospheric showers by antennas with the ability of these antennas to be phased towards a source to detect the radio signal of the atmospheric showers generated by gamma rays of very high energy from known sources, according to a process identical to that of UHECR. Unlike the case of UHECR, we then know where the signal should come from. The immediate advantage is the gain in detection sensitivity, which varies in direct proportion to the square root of the number of antennas involved. The other advantage is the possibility of reaching a useful observation cycle close to $100 \%$, since the day/night alternation and the weather have no influence on the detection itself (except in case of large local atmospheric electric fields during thunderstorms). Based on our experience of ultrafast radio transient detection and on the context of the Nançay radio astronomy station for the expertise in radio interferometry, we propose to explore the possibilities offered by this idea and to try for the first time to detect the radio signal produced by an ultra-high energy gamma air shower from an identified astrophysical source. It will be a question of knowing the expected value of the electric field produced by a shower initiated by such 
photons, or more generally the energy of the photon making it possible to produce a detectable electric field with regard to the limits of detection derived from our knowledge of cosmic rays radio signals. Given the energy threshold of detection found for the UHECR on observations with individual antennas (of the order of few $10^{16} \mathrm{eV}$ ), it is likely that this method applied to gamma should concern energies beyond the usual limits reached by current and future Cerenkov telescopes, from a few hundred $\mathrm{TeV}$ to a few tens $\mathrm{PeV}$ - provided astrophysical sources can produce gammas at such energies. But we think that this new detection principle deserves to be explored, because all the technical elements seem to be united to make it "explorable".

\section{The NenuFAR radio telescope}

CODALEMA is installed in a very rich instrumental environment (Fig. 1), since the Nançay radio astronomy station houses a set of radio telescopes covering wavelengths from decametre to decimetre (frequency range [10 - 3500] MHz). As many international developments aim to finalise the SKA project, a special effort is being made at the moment on the decametric domain, with the installation of the FR606 station of the international radio telescope LOFAR [8] and the construction, already well advanced, of the NenuFAR array ("New Extension in Nançay Upgrading LOFAR") [9].

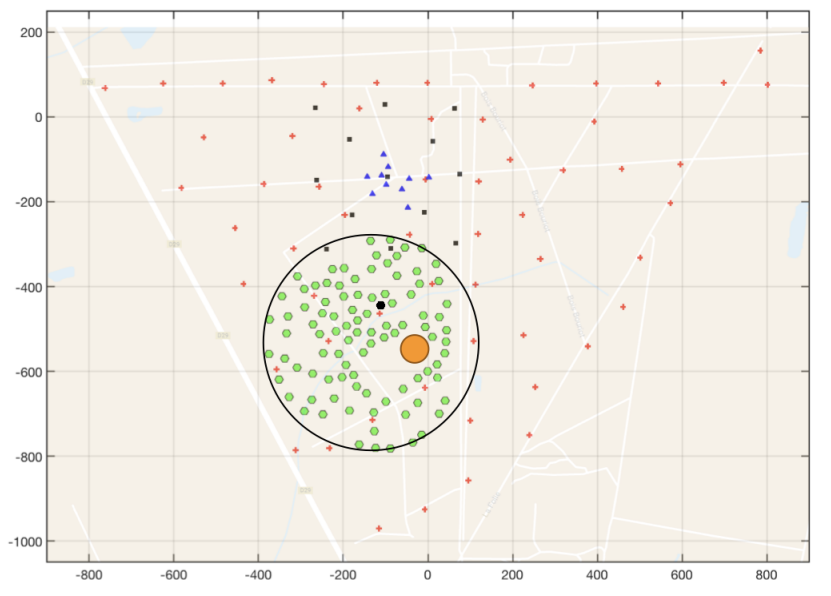

Figure 1: Map of the Nançay observatory, featuring some of the setups currently involved in the radio detection of EAS. Red crosses: CODALEMA autonomous stations, black squares: scintillators, blue triangles: compact array, green hexagons: NenuFAR mini-arrays (dark one is MA 12 , see text) into the $500 \mathrm{~m}$ diameter black circle. Orange disk: LOFAR FR606 LBA station. The area covered by CODALEMA is $\simeq 1.1 \mathrm{~km}^{2}$.

\subsection{Description of NenuFAR}

Also known as the LOFAR Super Station (LSS) in Nançay, NenuFAR is a digital radio telescope consisting - in the long term - of 1824 crossed-dipoles antennas arranged in 96 mini-arrays (hereafter $\mathrm{MA}_{19}$ ) of 19 antennas. In 2019, $56 \mathrm{MA}_{19}$ of those 96 are installed and operational. The antennas have a dual polarisation, along North West - South East (NWSE) and North East South West (NESW) directions respectively. They are identical to the ones of the CODALEMA Compact Array [7], themselves derived from the ones of the LWA telescope [10], on the aerial of which has been coupled the low-noise amplifier developed for CODALEMA [11]. The $96 \mathrm{MA}_{19}$ are distributed over an approximate circle of $500 \mathrm{~m}$ diameter $\left(0.2 \mathrm{~km}^{2}, \sim 7\right.$ LOFAR station diameter). In classical operation, each of the $\mathrm{MA}_{19}$ is analogically phased in any direction of the sky 
and in the whole or any sub-band of the [10-85] MHz frequency window, leading to an instantaneous sensitivity $\sqrt{19}$ times higher than that of a single antenna. The whole NenuFAR thus has an instantaneous sensitivity about twice the one of LOFAR's core, the "SuperTerp" which contains 7 individual LOFAR-LBA station ("Low Band Antenna", [30-80] MHz) of 96 single antennas.

NenuFAR has been designed to be used in two modes: as part of the LOFAR network in the LSS mode, and as a standalone instrument. Standalone measurements will be possible due to a dedicated receiver, allowing operation simultaneously with the LSS mode within the instantaneous field of view of the analog beam formed at the $\mathrm{MA}_{19}$ scale $\left(10\right.$ to $50^{\circ}$ from 10 to $\left.85 \mathrm{MHz}\right)$. Among the standalone observation modes, the use of a so-called "Transient Buffer Board" (TBB) system allows recording up to 5 seconds of the raw waveforms of all or part of the mini-arrays. In that case, the TBB are read on the decision made by an external trigger, without disrupting the current observations of the instrument. This capacity is particularly interesting for the observation of ultrabrief phenomena such as those produced by atmospheric showers. In the following, our proposal is to use NenuFAR in standalone mode with the TBB.

\subsection{NenuFAR as an UHECR detector}

NenuFAR and LOFAR606 are completely surrounded by the 57 radio detection stations of CODALEMA. This environment provides a unique opportunity to test and improve techniques for cosmic ray radio detection, and could act as a pathfinder for air shower detection with the SKA [12]. An external - either "hard" or "soft" - trigger input on the TBB is available to trigger NenuFAR by CODALEMA during regular astronomical operation (either from the particle detector or from the whole array of autonomous stations). It allows to read the TBB of the whole, analogically phased $\mathrm{MA}_{19}$ at the reception of a CODALEMA trigger. The main drawback is that the mini-arrays could be pointed in any direction not necessarily compatible with the direction of arrival of the cosmic ray shower, thus lowering the sensitivity. However, thanks to gain simulations of the $\mathrm{MA}_{19}$ in their pointing direction, this should make it possible to scale up the MA19 signals with respect to the regular CODALEMA antennas ones. This observing mode, based on the reading of a small part (10 $\mu$ s over $5 \mathrm{~s}$ ) of the TBB on the reception of a triggering signal coming from CODALEMA, is currently being implemented and tested on NenuFAR. We thus envision to test at first cosmic ray observations with NenuFAR to calibrate its signals on well-known cosmic ray event signals recorded by CODALEMA, in order to determine the way the gamma-ray trigger should be built.

\subsection{NenuFAR as a gamma ray detector}

As already mentioned, the specificity of the gamma-ray sources is that we know their position in the sky, thus the arrival direction of the high-energy photon, contrarily to the UHECR case. It is therefore possible to use one or several mini-arrays phased together in the direction of the source and use them as a trigger on transient events. As for the cosmic ray observation modes, we can take benefit of the TBB of the remaining $\mathrm{MA}_{19}$, which would be triggered by the trigger arrays, to roll-back in time and find the transient in their memories. Each $\mathrm{MA}_{19}$ would then be a sampling point of the shower footprint at ground, exactly as it is done in UHECR radio detection arrays with single antennas. This will help to know the electric field distribution and use it to recover the properties of the primary photon, notably its energy thanks to simulations. In principle, to 
reach the maximal sensitivity of detection and lower the detection threshold in energy, we would use all the available mini-arrays analogically phased individually on the source to build the trigger, by reading their signals in real time and decide whether or not there has been a transient in their phased combination. This however is at that time out of reach, due to the huge amount of data to be processed online: indeed, each $\mathrm{MA}_{19}$ delivers $400 \mathrm{MB} / \mathrm{s}$ per polarization, which should be taken at the level of the TBB, where the raw waveforms are still recorded and should be read and then combined again to sum all the signals. We then chose a different approach, based on the experience gained on CODALEMA. It is possible to split the signal from individual $\mathrm{MA}_{19}$ before they enter the acquisition chain, but after analog phasing at the level of one $\mathrm{MA}_{19}$. Each polarization thus behaves as the signal of a single antenna, and can feed one input channel of a dedicated electronics able to combine several of them. A classical but dedicated electronics board of NenuFAR, accepting 8 channels as input, can then be used to make digital phasing online and produce a trigger on a simple threshold level, the latter being subsequently used to trigger the TBB of all the $\mathrm{MA}_{19}$. This would limit the trigger sensitivity, since at most $8 \mathrm{MA}_{19}$ can participate on one polarization, but this would at first be the most efficient and elegant way to proceed. Another option would be to build a dedicated analog trigger board similar to the one of CODALEMA, but accepting more than two input channels. At that time both solutions are under study, the first stage being to determine how many $\mathrm{MA}_{19}$ are necessary to reach a sub-PeV sensitivity on detection.

\section{First results on cosmic ray air showers}

The first test that has been done was to split the signal of a single $\mathrm{MA}_{19}$ and to feed one of the input channels of the trigger board of a nearby CODALEMA station. In that respect, one of the polarization of the regular Butterfly antenna of the station is replaced by one polarization of the $\mathrm{MA}_{19}$, increasing the signal amplitude by a theoretical factor of 19 (if considered in the direction where the gains are maximum) and the signal to noise ratio (or detection sensitivity) by a factor of $\sqrt{19}$. This will allow comparisons to be made between the signal of a single antenna and the one of the $\mathrm{MA}_{19}$ on the same cosmic ray events. Provided that the latter's energy is reconstructible, an estimate of the gain in sensitivity detection and a first attempt of an energy calibration of the signal of a mini-array can be made and extrapolated to the expected energy of a gamma photon. We chose the $\mathrm{MA}_{19}$ numbered 12 (hereafter MA12), located close to the centre of the NenuFAR array, and the nearby CODALEMA autonomous station $20 \mathrm{~m}$ away (see Fig. 1). Fig. 2 shows an example of one of the cosmic ray signals recorded.

The amplitude of the raw signal of MA12 is about 13.5 times the one of the associated single antenna. For that event, the mini-array was pointed at the zenith, thus not in the direction of the event. Rescaling the signal by the theoretical gain of MA12 if it was pointed in that direction, the expected amplitude would have been 2.9 times larger, thus about 27 times the one of a single antenna that would have been in NESW polarization. Knowing the event energy reconstructed by CODALEMA of $5.910^{17} \mathrm{eV}$ [7], the direct proportionality between signal amplitude and shower energy, and the fact that the actual energy of this event is at least 1.5 order of magnitude above a single antenna energy detection threshold (about $410^{16} \mathrm{eV}$ ), this would suggest that a single miniarray has an energy detection threshold as low as $1.410^{15} \mathrm{eV}$. Moreover, simulations show that, at 

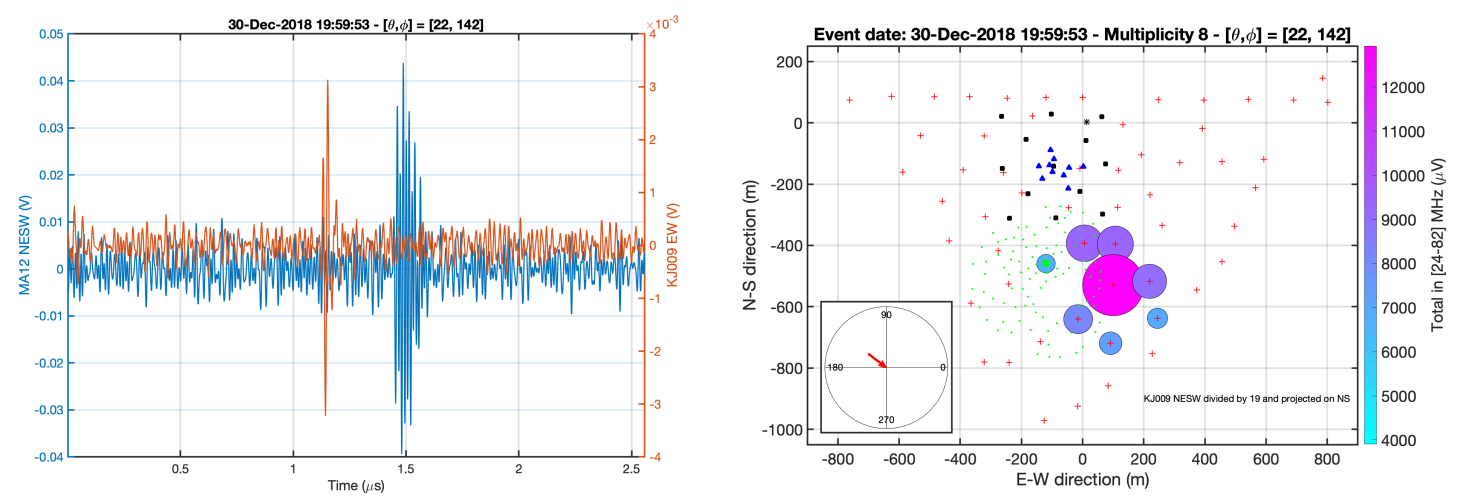

Figure 2: Left: an example of a cosmic ray signal recorded simultaneously by a CODALEMA EW antenna (orange Y scale, right) and the phased NESW polarization of MA12 (blue Y scale, left). The delay between the two pulses is due to the phasing system and the cable length. Right: the map of the event on the CODALEMA array, with a downscaling by a factor 19 of the signal of MA12 (indicated by the green dot) for visibility. Size and color of the circles feature the total amplitude of the signal recorded by each involved station. The direction of the event $\left(\theta=22^{\circ}\right.$, $\phi=142^{\circ}$ counted counterclockwise from East) is indicated by the arrow in the inset.

the location of MA12, for this event the electric field in NESW polarisation peaks at $22 \mu V \cdot m^{-1}$. With the gain correction factor of 2.9 to be applied, and considering that the galactic noise level is the same whatever is the number of antennas phased, the signal-to-noise ratio of this event would have been about 36, allowing us to think that the detection level of a pointed $\mathrm{MA}_{19}$ at $3 \sigma$ level should be around $1.8 \mu V \cdot \mathrm{m}^{-1}$, indeed corresponding to the peak electric field produced by a proton at $1.410^{15} \mathrm{eV}$ coming from the same direction. Of course, further processing is required for a precise estimate of the real detection threshold of a mini-array, and farther analysis will require fine simulations of the NenuFAR response, including a $\mathrm{MA}_{19}$ and combination of $\mathrm{MA}_{19}$ gains versus zenith and azimuth angles and electronics chain calibration, which is not done at present. However, the main conclusion is that this rough estimate allows us to believe that we can detect $\mathrm{PeV}$ gamma air showers with a single mini-array as a trigger for the rest of NenuFAR. If we apply a similar scaling factor by multiplying the number of mini-arrays phased together to be involved in the trigger, a gamma air shower with an energy of $100 \mathrm{TeV}$ should be accessible with at most 10 mini-arrays.

\section{Prospective on gamma ray observation - Estimate for a PeVatron}

The feature known as "the knee" in the cosmic ray spectrum (at $\sim 410^{15} \mathrm{eV}$ ), where the flux experiences a steepening, is interpreted as the beginning of the transition region where galactic sources disappear when extragalactic ones doesn't yet produce enough flux. It also implies the presence of so-called PeVatrons in our Galaxy, producing cosmic rays (mainly protons) at energies up to $10^{17} \mathrm{eV}$, which interact with the interstellar medium and produce gamma rays with an energy about ten times less than the one of the cosmic proton. Initiated by both gamma and cosmic ray observatories, as well as high energy neutrinos observatories such as IceCube, the search for those 
PeVatrons gave no direct results until the work from the H.E.S.S. collaboration [14] which show that such a PeVatron is present in the Galactic Centre, close to the central black hole Sgr A*. The direct detection of $\mathrm{PeV}$ photons by gamma ray observatories is hampered by the diffuse $\mathrm{TeV}$ gamma ray flux due to the interaction of cosmic rays with the dense gas cloud in the inner $200 \mathrm{pc}$ around the Galactic Centre, coming from nearby energetic sources. The detection of PeV gamma rays coming from this region would undoubtedly sign the presence of a PeVatron in our Galaxy. Unfortunately, due to the pointing limit in elevation $\left(20^{\circ}\right)$, the Galactic Centre is not observable by NenuFAR, though it appears low on the horizon at certain dates. It is however interesting to simulate a "fake" PeVatron such as the one expected to be hosted in the Galactic Centre, in order to know whether it could be observable from another location with a similar apparatus. Results of such simulations are presented on Fig. 3.
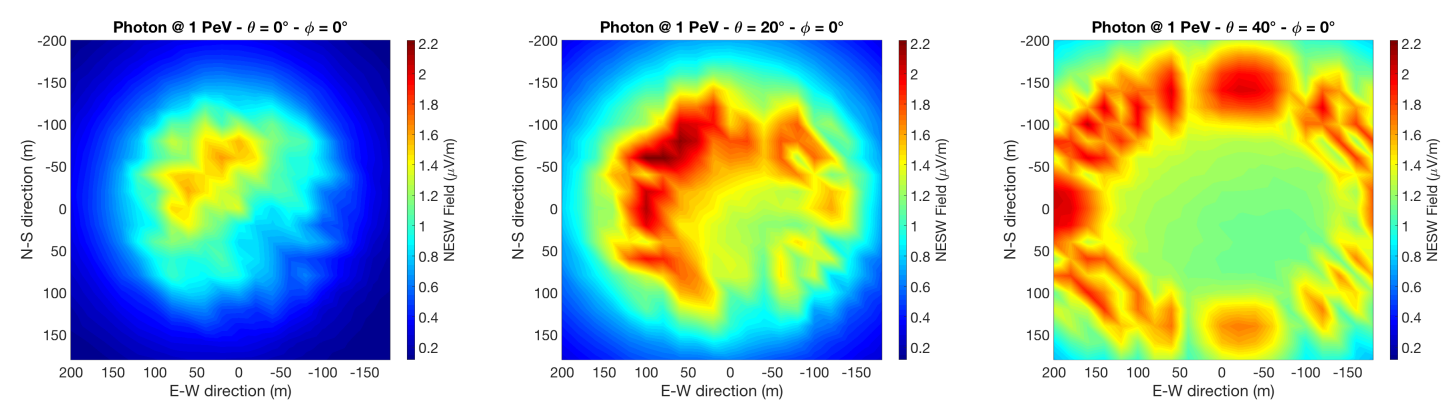

Figure 3: ZHAires simulations of a $1 \mathrm{PeV}$ photon shower coming from East at a zenith angle of 0,20 and $40^{\circ}$ (from left to right), in NE-SW polarization, observed in the [24-82] MHz band. The colour scale gives the electric field value in $\mu V \cdot \mathrm{m}^{-1}$. The Cerenkov ring is clearly visible with increasing zenith angle. The colour scale is the same for all the plots.

On the Cerenkov ring, the electric field reaches $2.2 \mu V \cdot \mathrm{m}^{-1}$, which is below the threshold for a single antenna but accessible for even a single mini-array $\left(>1.8 \mu \mathrm{V} . \mathrm{m}^{-1}\right)$, as stated in the previous section. Moreover, the footprint is quite different from the one of a proton or iron air shower, and its ground extension matches well with the area of NenuFAR. This makes the detection of PeV gamma highly feasible. Applying a scaling factor equal to the number of mini-arrays involved in the trigger, we can confirm that if the latter is made of $10 \mathrm{MA}_{19}$ a few hundreds $\mathrm{TeV} \gamma$ should be within reach of the instrument.

\section{Conclusion and outlook}

From the preliminary and rough estimations based on our experience, it appears that tens of $\mathrm{TeV}$ photons are difficult to detect with a reasonable configuration of NenuFAR, at least in a close future. We can not expect to detect gamma with energies under about $100 \mathrm{TeV}$. In that respect, if there are many extra-galactic sources of photons of the order of $1 \mathrm{TeV}$, however none of them are known as source of incident photons of the order of one hundred $\mathrm{TeV}$, due to their interaction with the cosmic microwave background on their trip to the Earth. We thus have to focus on galactic sources. If the first accessible source that we can think of is the Crab Nebula, now known to reach energies up to $450 \mathrm{TeV}$ [15] and almost always visible from Nançay, several other known galactic sources are in the field of view of NenuFAR. As soon as the trigger electronics mentioned 
in section 2.3 is ready, we will observe them during dedicated observing time with NenuFAR and CODALEMA. Several exploratory studies are still to do, notably the extrapolation of the number of expected photons above a given energy from the data obtained by Cerenkov experiments. In the direction of any gamma ray source, one can also expect to detect a certain number of cosmic rays above the NenuFAR threshold energy, whose signal should mimic the gamma shower signals. In principle, it should be quite easy to disentangle between gamma and UHECR, thanks to many factors: the electric field footprint will have a different extent and a different shape; for showers around $100 \mathrm{TeV}$, the muon contents of the UHECR shower is large, offering the possibility to trigger the nearby CODALEMA particle detector and use it as a veto; the use of simulations should also allow to recover the $X_{\max }$, thus the nature, of the primary. Around $1 \mathrm{PeV}$, we have shown that gamma photon air showers should be detectable and that their signature make them distinguishable from cosmic ray proton showers. It may open the way to a new gamma ray observatory, whose capabilities make it a multipurpose instrument (classical radio astronomy being still possible with the same telescope).

We acknowledge the Région Pays de la Loire for its financial support to the CODALEMA and EXTASIS programs. This work was supported by the Programme National des Hautes Energies of CNRS/INSU with INP and IN2P3, co-funded by CEA and CNES. This paper is based on data obtained using the NenuFAR radiotelescope. NenuFAR has benefitted from the following funding sources: CNRS-INSU, Observatoire de Paris, Station de Radioastronomie de Nançay, Observatoire des Sciences de l'Univers de la Région Centre, Région Centre-Val de Loire, Université d'Orléans, DIM-ACAV and DIM-ACAV+ de la Région Ile de France, Agence Nationale de la Recherche.

\section{References}

[1] T. Huege, Physics Reports 620 (2016), arXiv:1601.07426v1

[2] Frank G. Schroeder, Progress in Particle and Nuclear Physics, 93 (2017)

[3] T. Huege, M. Ludwig and C.W. James, AIP Conference Proceedings 1535128 (2012)

[4] J. Alvarez-Muñiz, W.R. Carvalho and E. Zas, Astropart. Phys. 35325 (2012)

[5] V. Marin and B. Revenu, Astropart. Phys. 35, p. $733-741$ (2012), arXiv:1203.5248 [astro-ph.HE]

[6] R. Dallier et al., EPJ Web Conf. 13501002 (2017), https://doi.org/10.1051/epjconf/201713501002

[7] A. Escudie et al., these proceedings (2019)

[8] M.P. van Haarlem et al. (LOFAR Collaboration), A\&A 556 A2 (2013)

[9] P. Zarka et al., [http://nenufar.obs-nancay.fr]

[10] http://lwa.unm.edu

[11] The Pierre Auger Collaboration, JINST 7, P10011 (2012)

[12] T. Huege et al., EPJ Web Conf. 13502003 (2017), https://doi.org/10.1051/epjconf/201713502003

[13] H.E.S.S. Collaboration, $A \& A$ 612, A1 (2018), https://doi.org/10.1051/0004-6361/201732098

[14] Abramowski et al, HESS collaboration, Nature 531, 476-479 (2016)

[15] M. Amenomori et al., accepted by PRL, arXiv:1906.05521 (2019) 